\title{
Consecutive Meals Planning by Using Permutation GA: The Case of Three Meal's Characteristics
}

\author{
Tomoko Kashima $^{*}$, Yukiko Orito ${ }^{\dagger}$, Hiroshi Someya ${ }^{\ddagger}$
}

\begin{abstract}
The consecutive meals planning is a combinatorial optimization problem that determines the meals plan on one period consisting of consecutive days. This paper designs two evaluation functions for the planning and applies a permutation GA to optimize it. The evaluation function measures the variation of appearance order of meals or meal's characteristics on the plan. In the numerical experiments, we show that our meals plan has a large variation of appearance order of meal's characteristics.
\end{abstract}

Keywords: Characteristics of Meal, Consecutive Meals Planning, Entropy, Permutation GA

\section{Introduction}

It is well known that the meals planning is a constrained combinatorial optimization problem that determines a food combination which minimizes or maximizes a given objective function. Many researchers have dealt with caloric intake minimization problem, cost minimization problem, and other problems under the constraints such as a nutritional requirement and the number of ingredients for their purposes such as healthy diet, balancing meals, and nutrient recommendation $[1,2,3]$. Most of their problems are viewed as integer or mixed-integer linear programming problems. Hence, we can find a high precision optimum solution by using a simplex method, an internal point method, or other optimization methods. On the other hand, there are many reports which analyze the features in recipes data on web sites as a big data and which develop the meals recommendation systems [4, 5, 6, 7]. However, most of those reports focus on the meals on a day for individuals.

In this paper, we focus on the meals on consecutive days for the educational institutions such as elementary school and preschool. The educational institutions provide the school meal every daily lunch. In each institution, the individual nutrition manager generally decides every meal on plan by his/her own policy. For planning meals, they have to keep some strict rules such as an amount of calories and cost. However, they can decide the meals plan freely under those rules. For this reason, they may consecutively provide the meals in similar characteristics every day though their institutions have to provide various meals in different characteristics such as food style, ingredient, and cooking method, for

* Kindai University, Japan

$\dagger$ Hiroshima University, Japan

* Tokai University, Japan 
growth of an infant or a child. Hence, we have a critical problem that the provided meals have similar characteristics though it is desired that various meals are provided.

In order to avoid this problem, in this paper, we design two evaluation functions which measure the variation of appearance order of meals or meal's characteristics on the plan. And then we apply a permutation GA to optimize this meals planning. In the numerical experiments, we show that the optimized meals plan has a large variation of appearance order of meals or meal's characteristics.

This paper is organized as follows: First, we define the consecutive meals plan in Section 2. We design the evaluation functions for the consecutive meals planning in Section 3. Section 4 describes the permutation GA as an optimization method. Section 5 presents the results of numerical experiments, and we conclude our discussion in Section 6.

\section{Consecutive Meals Plan}

Generally, one meal consists of one main dish and some side dishes, but we deal with only a main dish in this paper. In other word, we determine the main dishes plan as the meals plan on one period consisting of consecutive days.

We first define the following notations.

- $t$ : Time point $t(t=1, \cdots, T)$ on a period.

- $\mathbf{X}_{m}$ : Meal Class $\mathbf{X}_{m}(m=1, \cdots, M)$.

- $x_{i, j}$ : Item $j$ of Characteristics $i$ of Meal Class. That is $x_{i, j}\left(i=1, \cdots, I, j=1, \cdots,\left.J\right|_{i}\right)$.

- $\mathbf{Y}(t)$ : Meal Class provided at $t$.

- $y_{i, j}(t)$ : Item $j$ of Characteristics $i$ of Meal Class provided at $t$. That is $y_{i, j}(t)(i=$ $\left.1, \cdots, I, j=1, \cdots,\left.J\right|_{i}\right)$.

- Y: Meals plan consisting of $T$ variables. That is a solution, $\mathbf{Y}=\{\mathbf{Y}(1), \cdots, \mathbf{Y}(T)\}$.

In this paper, we classify the individual meals into one of Meal Classes according to the meal's characteristics of three categories; "food style", "ingredient", and "cooking method." Characteristics 1, the category of food style, consists of two items; "Japanese" and "Western." Characteristics 2, the category of ingredient, consists of two items; "Meat" and "Fish." Characteristics 3, the category of cooking method, consists of four items; "Simmer", "Fry", "Saute", and "Deep-fry."

Here, let $i(i=1, \cdots, I)$ be the kind of meal's characteristics. Let $\left.j\right|_{i}\left(j=1, \cdots,\left.J\right|_{i}\right)$ be the kind of items of Characteristics $i$. Let $x_{i, j}$ be the meal's characteristics of Item $j$ of 
Characteristics $i$. Using these settings, in this paper, Meal Classes are defined as,

$$
\begin{aligned}
& \mathbf{X}_{m}=\left\{x_{1, j}, x_{2, j}, x_{3, j}\right\}, \quad(m=1, \cdots, M), \\
& x_{1, j} \in\left\{\begin{array}{l}
\text { Japanese }(j=1) \\
\text { Western }(j=2)
\end{array},\right. \\
& x_{2, j} \in\left\{\begin{array}{ll}
\text { Meat } & (j=1) \\
\text { Fish } & (j=2)
\end{array},\right. \\
& x_{3, j} \in\left\{\begin{array}{ll}
\text { Simmer } & (j=1) \\
\text { Fry } & (j=2) \\
\text { Saute } & (j=3) \\
\text { Deep-fry } & (j=4)
\end{array},\right. \\
& m=\sum_{i=1}^{2}\left\{\left.\left(\left.j\right|_{i}-1\right) \prod_{k=i+1}^{3} J\right|_{k}\right\}+\left.j\right|_{3}, \quad\left(\left.j\right|_{i} \in\left\{1, \cdots,\left.J\right|_{i}\right\}\right), \\
& M=\left.\prod_{i=1}^{3} J\right|_{i}=2 \cdot 2 \cdot 4=16 .
\end{aligned}
$$

For example, The Meal Class $X_{1}=\left\{x_{1,1}, x_{2,1}, x_{3,1}\right\}$ is categorized by Japanese-style and simmered meat dish. The Meal Class $X_{16}=\left\{x_{1,2}, x_{2,2}, x_{3,4}\right\}$ is categorized by Western-style and deep-fried fish dish.

From the practical viewpoints, the educational institutions such as elementary school and preschool have many opportunities to provide a special meal on an event day. For example, Japanese preschool provides a chicken dish at Christmas. In a word, Meal Class provided at an event date is fixed on the meals plan. Hence, we assume that the event date which provides a special meal is randomly given on the period for the consecutive meals planning.

Here, let $t^{(e)}$ be the time point for event date and let $\mathbf{X}^{(e)}\left(\mathbf{X}^{(e)} \in\left\{\mathbf{X}_{1} \cdots, \mathbf{X}_{M}\right\}\right)$ be the Meal Class provided at $t^{(e)}$. The meals plan on the period consisting of $T$ time points is defined as,

$$
\begin{aligned}
& \mathbf{Y}=\{\mathbf{Y}(1), \cdots, \mathbf{Y}(T)\}, \\
& \mathbf{Y}(t)=\left.\left\{y_{1, j}, \cdots, y_{I, j}\right\}\right|_{t}, \quad(t=1, \cdots, T), \\
& \text { s.t. } \mathbf{Y}(t) \in\left\{\mathbf{X}_{1}, \cdots, \mathbf{X}_{M}\right\}, \quad \mathbf{Y}\left(t^{(e)}\right)=\mathbf{X}^{(e)}, \quad \mathbf{X}^{(e)} \in\left\{\mathbf{X}_{1} \cdots, \mathbf{X}_{M}\right\} .
\end{aligned}
$$

The meals plan defined by Equation (2) is the solution for the consecutive meals planning.

\section{Evaluation Functions for Consecutive Meals Planning}

It is well known that the information entropy is an index which measures the predictability/unpredictability of information content and is defined as the probability of observing event. In this paper, we design two evaluation functions for the consecutive meals planning by using the information entropy.

\subsection{Entropy of Meal Class}

We design an evaluation function which measures the variation of appearance order of Meal Classes on the meals plan. 
The information entropy of Meal Class is maximized if all Meal Classes $\left\{\mathbf{X}_{1}, \cdots, \mathbf{X}_{M}\right\}$ are observed the same number on the period. However, the information entropy has a problem that it cannot measure the order which the event occurs though it can measure the number of occurrences. For example, when there are two events, $E_{1}$ and $E_{2}$, on the period consisting of 4 time points $\left\{t_{1}, t_{2}, t_{3}, t_{4}\right\}$, the value of information entropy obtained from $\left\{E_{1}, E_{2}, E_{1}, E_{2}\right\}$ on $\left\{t_{1}, t_{2}, t_{3}, t_{4}\right\}$ is the same as that of $\left\{E_{1}, E_{1}, E_{2}, E_{2}\right\}$ on $\left\{t_{1}, t_{2}, t_{3}, t_{4}\right\}$.

Our evaluation function has to measure the variation of appearance order of Meal Classes on the meals plan. For measuring the appearance order, we divide one period consisting of $T$ time points into the short intervals consisting of the number of Meal Classes. As described in Equation (1), the number of Meal Classes is $M$. Here, the first interval consists of $M$ time points from $t=1$ to $t=M$. Moreover, the interval moves from the first one to the last one every one time point on the period. The last interval consists of $M$ time points from $t=T-M+1$ to $t=T$. Hence, we can divide the period into $T-M+1$ intervals for Meal Class.

It is expected that maximizing the information entropy on each of all intervals means maximizing the variation of appearance order of Meal Classes. Here, let $\alpha_{m}(t)$ be the binary variable 1/0 to which the appearance/non-appearance of Meal Class $\mathbf{X}_{m}$ at $t$ is expressed. Let $P_{m}$ be the frequency rate of $\mathbf{X}_{m}$ provided on an interval. For Meal Class $\mathbf{X}_{m}$, we design the evaluation function, "Entropy of Meal Class," as follows.

$$
\begin{aligned}
& L=-\frac{1}{T-M+1} \times\left(\sum_{t=1}^{T-M+1} \sum_{m=1}^{M} P_{m}(t) \log P_{m}(t)\right), \\
& P_{m}(t)=\frac{1}{M} \sum_{p=0}^{M-1} \alpha_{m}(t+p), \quad(t=1, \cdots, T-M+1, m=1, \cdots, M), \\
& \alpha_{m}(t)=\left\{\begin{array}{ll}
1 & \left(\mathbf{Y}(t)=\mathbf{X}_{m}\right) \\
0 & \left(\mathbf{Y}(t) \neq \mathbf{X}_{m}\right)
\end{array}, \quad(t=1, \cdots, T) .\right.
\end{aligned}
$$

On the other hand, for maximizing the variation of Meal Classes provided on the meals plan, it is desired that the number of each of all Meal Classes is the same. Hence, we define it as $T / M$ by using the length of period $T$ and the number of Meal Classes $M$. If $T$ is not a multiple of $M$, the number of each Meal Class has the maximum difference of 1 .

\subsection{Entropy of Meal's Characteristics}

The Entropy of Meal Class, $L$, measures the variation of appearance order only of Meal Classes on the meals plan. However, Meal Class is characterized by the combination of three meal's characteristics; food style, ingredient, and cooking method, in this paper. In this section, we design an evaluation function which measures the variation of appearance order of meal's characteristics of Meal Class.

For measuring the appearance order of meal's characteristics, we divide one period consisting of $T$ time points into the short intervals consisting of the number of items for each meal's characteristics. As described in Equation (1), the number of items of Characteristics $i$ is $\left.J\right|_{i}$. Here, the first interval consists of $\left.J\right|_{i}$ time points from $t=1$ to $t=\left.J\right|_{i}$. Moreover, the interval moves from the first one to the last one every one time point on the period. The last interval consists of $\left.J\right|_{i}$ time points from $t=T-\left.J\right|_{i}+1$ to $t=T$. Hence, we can divide the period into $T-\left.J\right|_{i}+1$ intervals for Characteristics $i$.

It is expected that maximizing the information entropy on each of all intervals means maximizing the variation of appearance order of meal's characteristics. Here, let $\alpha_{i, j}(t)$ 


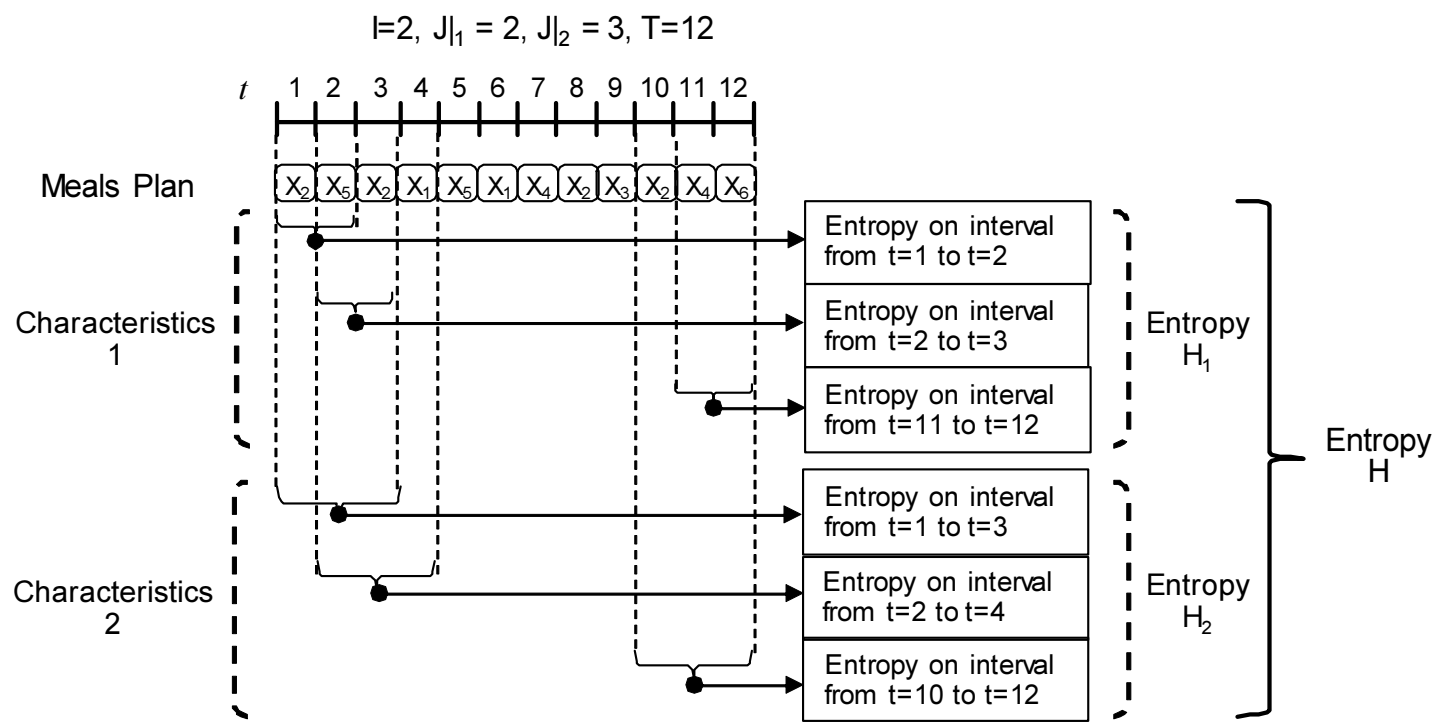

Figure 1: Entropy of Meal's Characteristics

be the binary variable $1 / 0$ to which the appearance/non-appearance of $x(i, j)$ (Item $j$ of Characteristics $i$ ) at $t$ is expressed. Let $P_{i, j}$ be the frequency rate of $x(i, j)$ provided on an interval. As with Equation (3), we design the evaluation function of meal's characteristics, "Entropy of Meal's Characteristics," as follows.

$$
\begin{aligned}
& H=\sum_{i=1}^{I} w_{i} \cdot H_{i}, \\
& H_{i}=-\frac{1}{T-\left.J\right|_{i}+1}\left(\sum_{t=1}^{T-\left.J\right|_{i}+1} \sum_{j=1}^{\left.J\right|_{i}} P_{i, j}(t) \log P_{i, j}(t)\right), \quad(i=1, \cdots, I), \\
& P_{i, j}(t)=\frac{1}{\left.J\right|_{i}} \sum_{p=0}^{\left.J\right|_{i}-1} \alpha_{i, j}(t+p), \quad\left(t=1, \cdots, T-\left.J\right|_{i}+1, j=1, \cdots,\left.J\right|_{i}\right), \\
& \alpha_{i, j}(t)=\left\{\begin{array}{rr}
1 & \left(y_{i, j}(t)=x_{i, j}\right) \\
0 & \left(y_{i, j}(t) \neq x_{i, j}\right)
\end{array}, \quad(t=1, \cdots, T),\right.
\end{aligned}
$$

where $w_{i}$ is a weighting coefficient of $H_{i}$ and is given in the numerical experiments.

Here, as an example, the Entropy of Meal's Characteristics in the case study of $T=12$ (Length of period), $I=2$ (The number of characteristics), $\left.J\right|_{1}=2$ (The number of items of Characteristics 1), and $\left.J\right|_{2}=3$ (The number of items of Characteristics 2) is shown in Figure 1.

In Figure 1, the meals plan consists of $T=12$ time points on the period. Each provided Meal Class is categorized into two characteristics. For Characteristics 1, the information entropy of two dates is calculated because the number of items is $\left.J\right|_{1}=2$, and thereby we obtain 11 information entropies and the average of 11 entropies, $H_{1}$. For Characteristics 2 , the information entropy of three dates is calculated because the number of items is $\left.J\right|_{2}=3$, and thereby we obtain 10 information entropies and $H_{2}$. Finally, we can obtain the Meal Entropy of Meal's Characteristics, $H$, by using $H_{1}$ and $H_{2}$. 


\section{Permutation GA}

In this paper, we apply a permutation GA to optimize the consecutive meals planning. The permutation GA expresses a round trip route most simply.

\subsection{Genetic Representation and Fitness Value}

In the genetic representation of the permutation GA, we replace a solution to an individual. The provided meals on a plan are represented by the integer sequence $\{v(1), \cdots, v(T)\}$. The individual consisting of the integer from $v(1)$ to $v(T)$ is denoted by,

$$
\begin{aligned}
& \mathbf{v}=\{v(1), \cdots, v(T)\}, \\
& \mathbf{v}(t)=w_{s} \bmod M, \quad w_{s} \in\{1, \cdots, T\}, \\
& w_{s_{1}} \neq w_{s_{2}}, \quad\left(s_{1}, s_{2}=1, \cdots, T, s_{1} \neq s_{2}\right) .
\end{aligned}
$$

Hence, at time point $t$, we have two kinds of variables, the variable $X_{m}$ of solution and the variable $v(t)$ of individual.

We employ the Entropy of Meal Class given by Equation (3) and the Entropy of Meal's Characteristics given by Equation (4) as the fitness values, respectively. The permutation GA tries to find the optimum solution which maximizes one of the fitness values.

\subsection{Genetic Operations}

The permutation GA generates the individuals in the initial population and makes new offspring by the crossover and the mutation. On each generation, the GA selects the individuals to the new generation by the elitism and tournament selections. Each operation of the permutation GA is designed as follows.

Note that Meal Classes provided at event date are randomly given on the period and are fixed on the procedure of permutation GA. They are not operated by the crossover and the mutation.

\section{Initial State}

On the first generation, the permutation GA generates $N_{p}$ individuals in the initial parents' population. Each individual consists of $T$ variables which are randomly selected from the integer sequence $\{1, \cdots, T\}$. Note that the Meal Classes provided at event date are fixed in advance.

\section{Evaluation and Selection}

The permutation GA applies the elitism and the tournament selections to select the individuals to the next population. First, let $E_{\text {rate }}$ be the elitist rate. The permutation GA selects $E_{\text {rate }} \times N_{p}$ individuals to the next population from the current population in high order of fitness value. Next, let TM_SIZE be the tournament size. The permutation GA selects $\left(1-E_{\text {rate }}\right) \times N_{p}$ individuals to the next population by selecting the best individual on each tournament group consisting of TM SIZE individuals.

\section{Crossover}

Let $P_{c}$ be the crossover rate. The permutation GA makes new $P_{c} \times N_{o}$ individuals by using the order crossover [8] for exchanging the partial structure between two individuals selected at random while keeping order of integer sequence. 
4. Mutation

Let $P_{m}$ be the mutation rate. The permutation GA makes new $P_{m} \times N_{o}$ individuals by exchanging two variables selected at random on one individual.

\section{Termination Criterion}

The permutation GA repeats the operations of producing the offspring population and performing the selection until the maximum number of the repetitions, $t_{\max }$, is satisfied.

From the last population, we choose one solution whose individual has the highest fitness value of all. This solution is the optimum or the quasi-optimum meals plan for the consecutive meals planning.

\section{Numerical Experiments}

We applied the permutation GA to optimize the consecutive meals planning. The experimental settings and the parameters are described in Section 5.1 and the results are shown in Section 5.2.

\subsection{Experimental Setting and Parameters}

In the numerical experiments, the characteristics in Meal Classes are categorized into each of food style, ingredient, and cooking method, $\left(x_{1, j}, x_{2, j}, x_{3, j}\right)$. Meal Class of experiments is defined by the same condition as Equation (1).

The setting for the consecutive meals planning is as follows.

- The number of Meal Classes: $M=16$ (given by Equation (1)).

- Length of period: $T=245$ (The number of weekdays in 2016).

- The number of event dates: 24 (Two days per one month).

- Weighting parameters for Entropy of Meal's Characteristics: $w_{1}=1, w_{2}=1$, and $w_{3}=1$.

Hence, the consecutive meals planning is the problem of determining the solution $\mathbf{Y}=$ $\{\mathbf{Y}(1), \cdots, \mathbf{Y}(245)\}$ such that the fitness value (Entropy of Meal Class or Entropy of Meal's Characteristics) is maximized under the condition that Meal Classes are randomly selected at the fixed 24 event dates on the period.

The parameters of the permutation GA are set as follows.

- Parents' Population Size: $N_{p}=100$.

- Offspring's Population Size: $N_{o}=200$.

- Crossover Rate: $P_{c}=0.9$.

- Mutation Rate: $P_{m}=0.1$.

- Elitist Rate: $E_{\text {rate }}=0.01$ (One individual per one generation).

- Tournament Size: $T M \_S I Z E=10$.

- The maximum number of the repetitions: $t_{\max }=1000$.

- Algorithm Run: 10. 
Table 1: Results in the case employing the Entropy of Meal Class $(L)$

\begin{tabular}{l|cccc|}
\hline Algorithm run & $L$ & $H_{1}$ & $H_{2}$ & $H_{3}$ \\
\hline 1 & 3.87228 & 0.48361 & 0.49180 & 1.43115 \\
2 & 3.86304 & 0.50410 & 0.47951 & 1.48518 \\
3 & 3.86902 & 0.57787 & 0.54918 & 1.45419 \\
4 & 3.85924 & 0.50000 & 0.55328 & 1.41719 \\
5 & 3.88261 & 0.45902 & 0.53279 & 1.48468 \\
6 & 3.86902 & 0.45492 & 0.48361 & 1.47407 \\
7 & 3.86739 & 0.51639 & 0.49180 & 1.46195 \\
8 & 3.86902 & 0.56967 & 0.52869 & 1.41747 \\
9 & 3.86196 & 0.45082 & 0.54918 & 1.45158 \\
10 & 3.87099 & 0.54508 & 0.51639 & 1.42499 \\
\hline Max. & 3.88261 & 0.57787 & 0.55328 & 1.48518 \\
Min. & 3.85924 & 0.45082 & 0.47951 & 1.41719 \\
Avg. & 3.86846 & 0.50615 & 0.51762 & 1.45025 \\
SD. & 0.00650 & 0.04626 & 0.02898 & 0.02645 \\
\hline
\end{tabular}

\subsection{Results and Discussion}

In order to demonstrate the power of the evaluation functions, the Entropy of Meal Class and the Entropy of Meal's Characteristics, we compare both these functions.

First, we apply the permutation GA employing the Entropy of Meal Class to optimize the consecutive meals planning. As the results of this case, the fitness value, $L$, of the optimized solution is shown in Table 1 . The entropy of each of Characteristics $i(i=1,2,3)$ of the solution, $H_{i}$, are also shown in Table 1 , respectively.

Next, we apply the permutation GA employing the Entropy of Meal's Characteristics to optimize the consecutive meals planning. As the results of this case, the fitness value, $H$, of the optimized solution is shown in Table 2. The entropy of each of Characteristics $i(i=1,2,3)$ of the solution, $H_{i}$, are also shown in Table 2 , respectively.

From Tables 1 and 2, the Entropy of Meal Class, $L$, is higher than the Entropy of Meal's Characteristics, $H$. However, it is impossible to compare $L$ with $H$ because $L$ consists only of the variation of Meal Classes and does not depend on the variation of meal's characteristics. On the other hand, all of $H_{i}(i=1,2,3)$ in the case employing the Entropy of Meal's Characteristics are higher than those of the case employing the Entropy of Meal Class. Moreover, the standard deviations of $H_{i}(i=1,2,3)$ in the case employing the Entropy of Meal's Characteristics are smaller than those of the case employing the Entropy of Meal Class. Hence, we conclude that the permutation GA employing Entropy of Meal's Characteristics can optimize the meals plan having a large variation of appearance order of meal's characteristics on the meals plan.

On the other hand, the standard deviation of $H$ s is larger than that of Ls. The Entropy of Meal Class, $L$, consists only of the variation of Meal Classes, but the Entropy of Meal's Characteristics, $H$, consists of three entropies, $H_{1}, H_{2}$, and $H_{3}$. The individual $H_{i}$ s have the trade-off relations for maximizing the variation of meal's characteristics because all of characteristics have the relation of mutual dependency in Meal Class. The consecutive meals planning for measuring the variation of appearance order of meal's characteristics can be viewed as one of the multi-objective optimization problems. We need to take a new idea which defeats such an evolutionary stagnation to the permutation GA. This is our future 
Table 2: Results in the case employing the Entropy of Meal's Characteristics $(H)$

\begin{tabular}{l|cccc}
\hline Algorithm run & $H$ & $H_{1}$ & $H_{2}$ & $H_{3}$ \\
\hline 1 & 3.49031 & 0.81148 & 0.86066 & 1.81818 \\
2 & 3.47998 & 0.82377 & 0.84836 & 1.80785 \\
3 & 3.50671 & 0.82377 & 0.86475 & 1.81818 \\
4 & 3.49011 & 0.84016 & 0.85656 & 1.79339 \\
5 & 3.46142 & 0.83607 & 0.83197 & 1.79339 \\
6 & 3.44919 & 0.81967 & 0.82787 & 1.80165 \\
7 & 3.50674 & 0.83197 & 0.85246 & 1.82231 \\
8 & 3.48415 & 0.81967 & 0.84836 & 1.81612 \\
9 & 3.44079 & 0.82377 & 0.84016 & 1.77686 \\
10 & 3.45925 & 0.84016 & 0.84016 & 1.77893 \\
\hline Max. & 3.50674 & 0.84016 & 0.86475 & 1.82231 \\
Min. & 3.44079 & 0.81148 & 0.82787 & 1.77686 \\
Avg. & 3.47687 & 0.82705 & 0.84713 & 1.80269 \\
SD. & 0.02314 & 0.00962 & 0.01207 & 0.01659 \\
\hline
\end{tabular}

work.

\section{Conclusions}

In the consecutive meals planning, we designed two evaluation functions. One measures the variation of appearance order of Meal Classes on the meals plan. The order measures the variation of appearance order of meal's characteristics on the meals plan. We applied the permutation GA employing the functions to optimize the planning.

In the numerical experiments, we showed that the permutation GA employing the Entropy of Meal's Characteristics is very useful for finding the good meals plan with a large variation of appearance order of meal's characteristics.

However, the evaluation function, the Entropy of Meal's Characteristics, has the tradeoff relations between the variables. We have to consider these relations. In addition, we need to quantify the quality of the meals plan obtained by the permutation GA. To show the advantages and the features in the meals plan obtained by the permutation GA, we will compare it with other plans obtained by other evolutionary algorithms and greedy methods such as hill climbing methods.

\section{Acknowledgment}

This work was supported by JSPS KAKENHI Grant Numbers \#25750007 and \#15K00339.

\section{References}

[1] L.M. Lancaster, "The History of the Application of Mathematical Programming to Menu Planning," European Journal of Operational Research, vol. 57, 1992, pp.339347.

[2] N. Darmon, E. Ferguson, and A. Briend, "Linear and Nonlinear Programming to Optimize the Nutrient Density of a Population's Diet: An Example Based on Diets of 
Preschool Children in Rural Malawi," American Society for Clinical Nutrition, vol. 75, no. 2, 2002, pp.245-253.

[3] D.D. SalooKolayi, A.T. Yansari, and S.H. Nasseri, "Application of Fuzzy Optimization in Diet Formulation," The Journal of Mathematics and Computer Science, vol. 2, no. 3, 2011, pp.459-468.

[4] D. Elsweiler and M. Harvey, "Towards Automatic Meal Plan Recommendations for Balanced Nutrition," Proceedings of the 9th ACM Conference on Recommender Systems, 2015, pp.313-316.

[5] C. Trattner and D. Elsweiler, "Investigating the Healthiness of Internet-Sourced Recipes: Implications for Meal Planning and Recommender Systems," Proceedings of the 26th International Conference on World Wide Web, 2017, pp.489-498.

[6] J.M. Cadenas, D.A. Pelta, H.R. Pelta, and J.L. Verdegay, "Application of Fuzzy Optimization to Diet Problems in Argentinean Farms," European Journal of Operational Research, vol. 158, 2004, pp.218-228.

[7] T. Kashima, S. Matsumoto, and H. Ishii, "Evaluation of Menu Planning Capability Based on Multi-dimensional 0/1 Knapsack Problem of Nutritional Management System," IAENG International Journal of Applied Mathematics, vol. 39, no.3, 2009, IJAM_39_3_04.

[8] L. Davis, "Applying Adaptive Algorithms to Epistatic Domains." Proceedings of the International Joint Conference on Artificial Intelligence, 1985, pp.162-164. 